\title{
A comparison of visual analog scale and Wong Baker facial pain scale for pain measurement in post lower caesarean section case
}

\author{
Neena Gupta, Sachidanand Gupta*, Apurva Agarwal, Shaily Agarwal, Rita Mahto
}

Department of Obstetrics and Gynaecology, GSVM Medical College, Kanpur, Uttar Pradesh, India

Received: 04 July 2016

Accepted: 05 August 2016

\section{*Correspondence:}

Dr. Sachidanand Gupta,

E-mail: drsngupta2000@gmail.com

Copyright: (c) the author(s), publisher and licensee Medip Academy. This is an open-access article distributed under the terms of the Creative Commons Attribution Non-Commercial License, which permits unrestricted non-commercial use, distribution, and reproduction in any medium, provided the original work is properly cited.

\section{ABSTRACT}

Background: There is various pain measurement tools commonly used to measure pain intensity. However, no single standard exists for quantifying pain in post caesarean section. In our study, we have divided two groups according to use of pain scales and found one scale is more useful than the other in the post caesarean cases.

Methods: The study has been conducted in upper India Sugar Exchange Maternity Hospital department of obstetrics and gynaecology, GSVM Medical College, Kanpur for the duration of 18 months starting from February 2014 to August 2015 on 100 women who were operated for lower caesarean section (LSCS) under spinal anesthesia. Women were divided into two groups according to pain scales visual analog scale (VAS) and Wong Baker facial pain scale (WBFPS). All these selected women were studied in details with regards to clinical history, general examination and local examination. Women from respective group were asked to rate their pain intensity on these pain scales. SPSS was used to analyze the data statistically.

Results: Using analysis of variance, we found that there is significant relationship within the groups having different parameters at different time interval at different probability level. There is a mutual relationship between the groups at different probability level.

Conclusions: The study shows that VAS is the most sensitive and reliable tool to measure post LSCS pain as compared to WBFPS and minimum consumption of rescue analgesia in VAS group to maintain minimum pain score to keep the patient pain free.

Keywords: Pain, Lower caesarean section, Visual analog scale, Wong baker facial pain scale

\section{INTRODUCTION}

Precise and systematic pain assessment is essential for correct diagnosis and determination of the most efficacious treatment plan for patients presenting with pain. This requires a valid and reliable tool to estimate the pain. Unlike other vital signs, pain is subjective on the part of both the patient and the healthcare professional. ${ }^{1}$ It is unacceptable for patients to experience unmanaged pain or for doctors/nurses have inadequate knowledge about pain and a poor understanding of their professional accountability in this aspect of care. ${ }^{2}$

In most of the Country pain is now considered the fifth vital sign. Pain rating scales have an essential place in clinical practice. Evidence suggests that pain rating scales are used as a way for patients to communicate their experience of pain and their response to treatment. ${ }^{3}$

There are many pain scales available to measure the pain of patients and its uses depends upon various factors such as age, language skill, and medical history. Visual analogue scale (VAS), the numerical rating scale (NRS), Wong Baker facial pain scale (WBFPS), descriptive pain scale (DPS) are the widely used pain scale. Pain is individual and subjective and modulated by several factors such psychological, physiological, age, education, culture, previous events, fear, anxiety and prognosis. Caesarean births differ from other major laparotomies because women are expected to recover expeditiously and 
take care for their new-borns within a few hours following the operation and this may affect the perceptions of pain and wellbeing. ${ }^{4}$

This study has been carried out to investigate the pain scoring systems in first time operated post lower segment caesarean section case to find sensitivity correlation in pain measuring scales such as VAS and WBFPS.

\section{METHODS}

This study was conducted in the department of obstetrics and gynecology of upper India sugar exchange maternity hospital, GSVM Medical College, Kanpur, from February 2014 to August 2015 on 100 numbers of Post LSCS women with primary caesarean section done under spinal anesthesia.

All these selected women were studied in details with regard to clinical history, general examination, and local examination for 72 hours in the postoperative period. Once patients consented, they were asked to rate their pain intensity by using two different pain scales, the VAS and Wong Baker facial pain scale. Patients were given a visual analog scale, a $10 \mathrm{~cm}$ line with anchor points of "no pain" and "worst imaginable pain", and asked to rate their pain intensity at the time of questioning by marking a line perpendicular to the VAS. The patient was also given WBFPS and we observe the patient's expression and given marks for her pain scoring. The order of the presentation of the VAS and WBFPS was randomized. As approximately equal no of patients asked by VAS and WBFPS within one minute interval. According to Bijur, the level of pain intensity is assumed not to change during a one-minute interval. ${ }^{5}$ Patient demographics such as age, gender, location and cause of pain were collected from the patient.

The goal of the proposed study was to focus on the limitations of that study by including lower levels of pain as well has high levels of pain. For the primary purpose of the study the independent variables include the visual analog scale and Wong Baker facial pain scale. The variables were operationalized by the personal experience of pain as reported by the participant and other variables measured by us.

Reliability was ensured as we used the same script to verbally obtain the patients' WBFPS and VAS score the same way every time. The same ruler was used to measure every participant.

All data were entered into Microsoft Excel and exported into SPSS for statistical analysis. To compare the correlation between the VAS and WBFPS, to analyze the VAS and WBFPS an independent samples t-test was utilized.

\section{RESULTS}

Using student t-test for testing the significant difference among different pain scales by measuring different parameters, we found relatively significant relationship between different groups at different level of significance. There is no significant relationship in between VAS and WBFPS $(\mathrm{t}=1.63, \mathrm{p}$-value >0.05) when taking blood pressure as a variable parameter (Table 1). VAS also shows the highly significant relation with pulse rate as compared to WBFPS $(\mathrm{t}=4$. 34 , $\mathrm{p}$ value<0.001) (Table 2). VAS and WBFPS shows nonsignificant role when respiratory rate was variable $(\mathrm{t}=0$. 24 p-value $>0.05$ (Table 3 ). VAS is more reliable than WBFPS for measuring pain intensity (t-2.54, pvalue<0.05) (Table 4).

Table 1: Comparison of different groups (having different parameter as blood pressure).

\begin{tabular}{|llll|}
\hline Groups & $(\mathrm{B})$ & $(\mathrm{C})$ & \\
\hline Parameters & BPVAS & BP WBFPS \\
\hline$(\mathrm{N}=100)$ & $(\mathrm{n}=50)$ & $(\mathrm{n}=50)$ & \\
\hline Mean & 71.80 & 71.66 & \\
\hline \pm SD & 0.38 & 0.47 & \\
\hline Groups & $\mathbf{t}$ & $\mathbf{p}$ & Interference \\
\hline $\begin{array}{l}\text { VAS versus } \\
\text { WBFPS }\end{array}$ & 0.62 & $>0.05$ & Non-significant \\
\hline
\end{tabular}

There is non-significant relationship which shows that no effect of BP variation seen in different groups (scales).

Table 2: Significant relationship between different groups (having parameter as pulse rate).

\begin{tabular}{|llll|}
\hline Groups & $(\mathrm{B})$ & $(\mathrm{C})$ \\
\hline Parameters & PRVAS & PRWBFPS \\
\hline$(\mathrm{N}=100)$ & $(\mathrm{n}=50)$ & $(\mathrm{n}=50)$ & \\
\hline Mean & 88.04 & 84.54 & \\
\hline$\pm \mathrm{SD}$ & 3.57 & 4.44 & \\
\hline Groups & $\mathbf{t}$ & $\mathbf{p}$ & Interference \\
\hline $\begin{array}{l}\text { VAS versus } \\
\text { WBFPS }\end{array}$ & 4.34 & $<0.001$ & Highly significant \\
\hline
\end{tabular}

The above table shows that there is a highly significant relationship in VAS in comparison to WBFPS corresponding to changes in pulse rate.

Table 3: Significant relationship between the groups (having different parameter as respiratory rate).

\begin{tabular}{|llll|}
\hline Groups & $(\mathrm{B})$ & $(\mathrm{C})$ & \\
\hline Parameters & PRVAS & RRWBFPS \\
\hline$(\mathrm{N}=100)$ & $(\mathrm{n}=50)$ & $(\mathrm{n}=50)$ & \\
\hline Mean & 18.53 & 18.62 & \\
\hline \pm SD & 1.28 & 1.59 & \\
\hline Groups & $\mathbf{t}$ & $\mathbf{p}$ & Interference \\
\hline $\begin{array}{l}\text { VAS versus } \\
\text { WBFPS }\end{array}$ & 0.24 & $>0.05$ & Non-significant \\
\hline
\end{tabular}

The above table shows significant relationship i.e. VAS is significant changes with the changes in respiratory rate as compared to WBFPS. 
It can be observed from Table 5, use of rescue doses of tramadol i.v injection shows the significant role of the VAS as compared to WBFPS. $(\mathrm{t}=2.23$, $\mathrm{p}$-value $<0.05)$ in post-caesarean case. The mean score of VAS on day 1 day 2 and day 3 of LSCS was found at lower side as compared to WBFPS (Figure 1). There is a minimum requirement of rescue doses in VAS as compared to WBFPS (Figure 2).

\section{DISCUSSION}

One hundred were approached and data were collected. Out of the 100 patients that consented, 50 were asked the VAS first and 50 patient asked for Wong Baker facial pain scale. $50 \%$ primigravida and $50 \%$ multi gravida patients were selected to equalize distribution for better comparison. Maximum numbers of patients (47.89\%) are lying between the age group of 21 to 30 years and belong to low socioeconomic condition $(46.66 \%)$ having the only primary level of education.

Table 4: Correlation among the pain scales.

\begin{tabular}{|llll|}
\hline Parameters & VAS score & WBFPS score \\
\hline$(\mathrm{N}=100)$ & $(\mathrm{n}=50)$ & $(\mathrm{n}=50)$ & \\
\hline Mean & 3.42 & 4.10 & \\
\hline \pm SD & 1.29 & 1.38 & \\
\hline Groups & $\mathbf{t}$ & $\mathbf{p}$ & Interference \\
\hline $\begin{array}{l}\text { VAS versus } \\
\text { WBFPS }\end{array}$ & 2.54 & $>0.05$ & Significant \\
\hline
\end{tabular}

The above table shows a significant relationship between VAS and WBFPS and VAS is a better measuring tool as compared to WBFPS.

Table 5: Relationship between rescue doses on different days in different groups.

\begin{tabular}{|c|c|c|c|c|c|c|c|c|}
\hline \multirow{2}{*}{ Groups } & \multicolumn{2}{|c|}{ Rescue analgesic (D1) } & \multicolumn{2}{|c|}{ Rescue analgesic (D2) } & \multicolumn{2}{|c|}{ Rescue analgesic (D3) } & \multicolumn{2}{|l|}{ Mean } \\
\hline & Mean & \pm SD & Mean & \pm SD & Mean & $\pm \mathrm{SD}$ & Mean & \pm SD \\
\hline VAS & 1.64 & 0.79 & 0.40 & 0.53 & - & - & 0.76 & 0.63 \\
\hline WBFPS & 1.24 & 0.90 & 0.28 & 0.44 & - & - & 0.90 & 0.61 \\
\hline Groups & t & $\mathbf{p}$ & Interfe & & & & & \\
\hline $\begin{array}{l}\text { VAS versus } \\
\text { WBFPS }\end{array}$ & 2.54 & $>0.05$ & Signifi & & & & & \\
\hline
\end{tabular}

The above table shows there is an insignificant role of WBFPS to maintain minimum pain score by using minimum rescue doses.

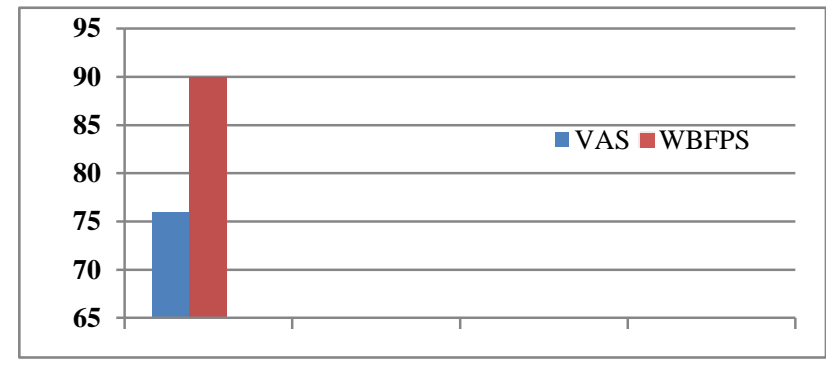

Figure 1: Requirement of rescue doses by using different pain scores.

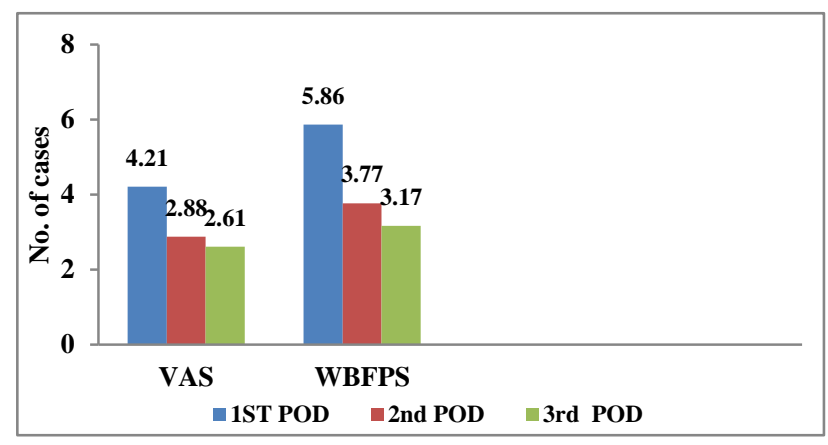

Figure 2: Mean scores of different pain scales on day 1 , day 2 and day 3.
In this study, parametric statistical tests were used to compare the mean of the VAS and the WBFPS and found the mean are correlative which is in line with the finding of Jaywant SS and Pai AV. ${ }^{6}$ In our study, we found nonsignificant relationship of blood pressure variation on different pain scale. Pulse rate variation is highly significant with WBFPS (t-4.34, $\mathrm{p}<0.001)$. As far as sensitivity is concerned, we found both scales sensitive, but VAS is the most sensitive and preferred scale among the study population over WBFPS. However, in the study of Mohammed et al. ${ }^{7}$ WBFPS was found most simple and preferred pain rating scale among the patients of osteoarthritis of north Telangana tribal district tertiary care center in Andhra Pradesh Region.

By measuring pain intensity in post-caesarean case use of rescue analgesic and regular analgesic approximately equally required in the VAS group as compared to WBFPS to keep the patient pain free.

This study shows that there is a correlation between the VAS and WBFPS when assessing pain in post-caesarean cases as previous ones. Although, the VAS is interval level data and WBFPS is qualitative data comparing the mean of these groups prove a correlation between the scales. 
We assume that patients are honest regarding their pain level and their pain can be objectified. Patients had dissimilarity in pain tolerances which may have caused a bias in the results. Moreover, Negative and positive feelings coexist during birth. ${ }^{8}$ Even though the simplicity of use was considered an advantage of the VAS, various patients misunderstood how to use it properly. VAS needed extra instruction during their pain assessment which may have skewed their pain result. Patients were required to provide written consent and therefore were aware of the research study, which may have caused a Hawthorne effect.

This study validates that both VAS and WBFPS are valid and reliable scale to measure pain when used to assess pain in post caesarean cases. However, it has been found that VAS is easy to administer verbally in a clinical setting, even among low level education and rural background patients. The study shows that VAS is valid and reliable scale and has a significant relation with different parameter such as blood pressure, pulse rate and respiratory rate and require minimum rescue dose of drugs to maintain minimum pain score for better management of pain and avoidance of unnecessary use of drugs compare to WBFPS. The different pain scales may be used for different group of patients but the preferred scale should be used consistently to get more valid results. $^{6}$

\section{CONCLUSION}

The study shows that VAS is the most sensitive and reliable tool to measure post LSCS pain as compared to WBFPS and minimum consumption of rescue analgesia in VAS group to maintain minimum pain score to keep the patient pain free.

\section{Funding: No funding sources Conflict of interest: None declared \\ Ethical approval: Not required}

\section{REFERENCES}

1. Hartrick CT, Kovan JP, Shapiro S. The numeric rating scale for clinical pain measurement: a ratio measure pain practice. 2003;3(4):310-6.

2. Diamond B. Legal aspects of pain management. Salisbury. Quay Books; 2002.

3. Williamson A, Hoggart B. Pain: a review of three commonly used pain rating scales. Journal of Clinical Nursing. 2005;14(7):798-804.

4. Karlström R. Engström-Olofsson K. Norbergh M. Sjöling, Hildingsson I. Postoperative pain after cesarean birth affects breastfeeding and infant care. Journal of Obstetric Gynecologic and Neonatal Nursing. 2007;36(5):430-40.

5. Bijur PE, Silver W, Gallagher EJ. Reliability of the visual analog scale for measurement of acute pain. Academic Emergency Medicine. 2001;8(12):1153-7.

6. Jaywant SS, Pai AV. A comparative study of pain measurement scales in acute burn patients. Indian Journal of Occupational Therapy. 2003;XXXV:3.

7. Bashir MS, Khade A, Borkar P, Saleem M, Lingaswamy V, Reddy D. A comparative study between different pain rating Scales in patients with osteoarthritis. Indian J Physiol Pharmacol. 2013;57(2):205-8.

8. Waldenström U, Borg IM, Olsson B, Sköld M, Wall S. The childbirth experience: a study of 295 new mothers. Birth. 1996;23:144-53.

Cite this article as: Gupta N, Gupta S, Agarwal A, Agarwal S, Mahto R. A comparison of visual analog scale and Wong Baker facial pain scale for pain measurement in post lower caesarean section case. Int J Reprod Contracept Obstet Gynecol 2016;5:3032-5. 